\title{
A TEORIA DA MENTE DE CRIANÇAS COM AUTISMO NA ÓTICA PIAGETIANA
}

\section{THE THEORY OF MIND OF CHILDREN WITH AUTISM IN THE PIAGETIAN PERSPECTIVE}

Patricia Lorena Gonçalves

E-mailpara correspondência: patricialorena@usp.br

Maria Thereza da Costa Coelho de Souza

\section{RESUMO}

Este artigo se refere a um estudo clínico do tipo exploratório que pretendeu investigar a relação entre as dificuldades de atribuição de estados mentais de crianças com autismo e as principais características do pensamento egocêntrico descrito por Jean Piaget. Para tanto, foi utilizado o método de estudo de casos de dois participantes diagnosticados com autismo, ambos com a idade de dez anos. Os procedimentos desta pesquisa foram: uma entrevista clínica com método piagetiano feita com os participantes; narrativas feitas pela pesquisadora de contos de fada e histórias sociais aos participantes; aplicação nestes da tarefa de crença 
falsa de Sally e Ann de Baron-Cohen, Leslie e Frith (1985) e análise do emprego de termos mentais e do discurso destes. O trabalho concluiu que crianças com autismo, apesar de poderem empregar termos mentais em seus discursos, geralmente, têm dificuldades de diferenciar entre o psicológico e o físico, apresentando tendência à representação imagética em detrimento da representação conceitual; desinteresse em comprovar o que afirmam e dificuldades em diferenciar o próprio ponto de vista do ponto de vista do outro, o que pode estar relacionado com suas dificuldades de atribuição de estados mentais.

Palavras-chave: Teoria da Mente; Autismo; Alteridade.

\section{ABSTRACT}

This article refers to a theoretical and clinical study of the exploratory type that aimed to investigate the relationship between the assignment difficulties of mental states of children with autism and the main features of egocentric thought described by Jean Piaget. For this, it was used the method of case studies of two participants diagnosed with autism, both at the age of ten years. The procedures in this study were: a clinical interview with Piaget's method made with the participants; narratives of fairy tales and social stories to the participants made by the researcher; application of false belief task of Sally and Ann (Baron-Cohen, Leslie e Frith, 1985 ) in participants; analysis of the employment of mental expressions and analysis of his speeches. This study concluded that children with autism, although they may employ of mental expression in his speeches, often have difficulties to differentiate between psychological and physical, with a tendency to image representation at the expense of conceptual representation, unwillingness to prove what they say and difficulties in differentiate their own point of view from the other ones, which may be related to their difficulties in attribution of mental states.

Keywords: Theory of mind; Autism; Otherness.

\section{INTRODUÇÃO}

Este artigo trata de uma pesquisa de mestrado que teve por objetivo investigar a relação entre as dificuldades de atribuição de estados mentais - ou como é conhecida atualmente, teoria da mente -, de crianças com autismo e a tendência destas ao pensamento egocêntrico (descrito por Jean Piaget).

Desde o estudo seminal de Kanner (1943) as publicações científicas em torno do tema do autismo têm se avolumado com o passar dos anos. Da mesma forma, são variadas as vertentes que buscam compreender esse fenômeno como as teorias psicanalíticas, a teoria afetiva, as neurociências, as teorias cognitivistas (coerência central, função executiva e teoria da mente), entre outras. Dada a amplitude 
de abordagens sobre este transtorno, procuramos nesta pesquisa ancorarmo-nos nas teorias cognitivistas, em especial, a respeito da teoria da mente e na epistemologia genética de Piaget.

Leo Kanner publicou em 1943 o artigo "Autistic disturbances of affective contact" na revista Nervous Children, referente à sua pesquisa com método de estudos de casos em que participaram onze crianças com idades entre dois e onze anos, sendo oito meninos e três meninas. Neste trabalho, Kanner (1943) relatou que desde 1938 tomara conhecimento de algumas crianças com características peculiares fascinantes e excepcionalmente diferentes de tudo o que havia sido descrito até então. As características observadas nos onze estudos de casos realizados por Kanner (1943) apontavam um isolamento extremo, um desejo obsessivo pela mesmice, dificuldades em adquirir linguagem verbal e que tinham em geral excelente memória. Assim, Kanner (1943) concluiu que talvez estas crianças viessem ao mundo com uma incapacidade inata para o contato afetivo, da mesma maneira que outras crianças nasciam com deficiências físicas ou intelectuais.

Inicialmente os sintomas do autismo foram comparados aos sintomas da esquizofrenia. Porém, diferenças qualitativas entre o autismo e as psicoses estudadas na época foram observadas nestes pacientes, como busca intermitente por imutabilidade, reação de horror frente a barulhos de volume alto, ecolalias etc. Estas diferenças contribuíram para a migração da terminologia "autístico" para “o autismo", uma nova patognomia (Laurent, 2014; Leon, 2002; Velloso, 2012).

A noção de autismo tem sofrido diversas alterações ao longo do tempo, contribuindo, de certa forma, para um número significativo de diagnósticos imprecisos. Lima (2010) esclarece que nos primeiros vinte anos, o autismo permaneceu imerso nas concepções psicodinâmicas, o que pode ter influenciado a hipótese de Kanner (1943) sobre uma possível relação entre os sintomas do autismo e as características peculiares dos pais das onze crianças analisadas, como frieza emocional no que diz respeito ao relacionamento com seus filhos e intelectualismo exacerbado.

As concepções psicodinâmicas passaram a dividir terreno com as concepções cognitivas a respeito do autismo a partir de meados dos anos 1960, principalmente depois da publicação do livro Infantile Autism, de Bernard Rimland (1964), que defendia ser o autismo, uma disfunção cognitiva e não afetiva. A partir dos anos 1980, influenciados pela revolução cognitivista nos preceitos psicológicos, boa parte dos estudiosos do autismo passou a considerá-lo como uma disfunção cognitiva de natureza neurológica, influenciada, principalmente, por fatores genéticos (Lima, 2010).

Embora as inúmeras controvérsias sobre a etiologia e definição do autismo tenham marcado as sete décadas que sucederam a sua primeira descrição feita por 
Kanner (1943), existe um consenso entre os estudiosos do tema em relação aos sintomas que o definem: prejuízos na interação social; consideráveis dificuldades na comunicação e interesses restritos e estereotipados (Assumpção Jr. \& Kuczynski, 2011; Dourado, 2011; Klin, 2006; Schwartzman, 2011).

O enfoque cognitivista a respeito do autismo tem possibilitado condições para formular teorias que buscam explicar sua sintomatologia, dentre elas, a teoria da mente (Amorim, 2008; Bosa \& Callias, 2000; Leon, 2002; Velloso, 2012).

A teoria da mente tem sido denominada pelos seus estudiosos como a capacidade de atribuir estados mentais (crenças, desejos, intenções e emoções) a si e aos outros, com o intuito de explicar, interpretar e predizer comportamentos. Segundo alguns autores, a aquisição de uma teoria da mente é imprescindível para a eficácia dos relacionamentos sociais, uma vez que auxilia a criança a antecipar suas ações, justamente por compreender e identificar as emoções e crenças do outro (Domingues, 2006; Golleman, 2006).

Os psicólogos Perner e Wimmer (1983), baseados no estudo de Premack e Woodrulf (1978) que originou o termo "teoria da mente", criaram a primeira tarefa de crença falsa. Com base no estudo de Perner e Wimmer (1983), Baron-Cohen, Leslie e Frith (1985) construíram a tarefa de crença falsa "Sally e Ann". A tarefa constitui-se no seguinte problema: Sally e Ann brincavam de bola e ao término da brincadeira, Sally guardou a bola em uma cesta, saindo da presença de Ann. Nesse momento, Ann retirou a bola da cesta e a colocou em uma caixa, sem que Sally tenha visto esta alteração de lugar. Pergunta-se ao examinando: "Quando Sally retornar, onde ela procurará a bola?".

$\mathrm{O}$ acerto nas tarefas de crença falsa demonstra que a criança tenha adquirido a teoria da mente por alguns motivos: em primeiro lugar a criança precisou deixar de levar em conta seu próprio ponto de vista (ela sabe que a bola está na caixa) para inferir sobre um ponto de vista diferente (o de Sally, que acredita que a bola está na cesta, pois não viu a troca da bola de lugar (Carraro, 2003).

Além da resolução das tarefas de crença falsa que mensuram a teoria da mente, a análise do emprego de termos mentais e a brincadeira de faz de conta tem sido alvo de escrutínio entre os teóricos (Leslie, 1987, 1988; Lillard, 1993) como possíveis indicadores de uma teoria da mente na criança.

Alguns estudiosos da teoria da mente (Jou \& Sperb, 1999; Perner, 1991; Rodrigues, 2005) têm afirmado que a habilidade de inferir estados mentais alheios só é possível de ser desenvolvida quando a criança é capaz de alcançar um nível de representação mental que a habilite a diferenciar entre o que é subjetivo do que é objetivo. Sendo assim, a partir desta capacidade de interpretar o mundo interno e o mundo externo, a criança torna-se cada vez mais sociável, passando a inferir sobre crenças, desejos e emoções alheias. 
Piaget (1947) advoga que a criança alcança um nível de representação mental que lhe permite diferenciar entre o que é subjetivo do que é objetivo quando inicia a superação do pensamento egocêntrico, pois enquanto a criança pensar que todos pensam como ela, não encontrará motivos para se conformar às verdades comuns, muito menos buscar comprovações lógicas sobre aquilo que acredita. Para este autor, "a lógica se desenvolve em função da socialização do pensamento" (Piaget, 1947, p. 33).

Para Piaget (1923), o pensamento egocêntrico é definido como um estágio primitivo do pensamento lógico e é marcado por três principais características que os distinguem entre si: inconsciência da diferenciação entre o próprio ponto de vista e o ponto de vista de outra pessoa; ausência de lógica, pois tende a afirmar o tempo todo a sua própria realidade; e tendência ao predomínio da representação imagética sobre a representação conceitual.

Podendo ser pensado como um transtorno global do desenvolvimento, o autismo infantil - que é compreendido na forma de um espectro entre alguns estudiosos (Assumpção Jr. \& Kuczynski, 2011; Dourado, 2011; Schwartzman, 2011) -, pode assim evidenciar atrasos ou o não desenvolvimento de estruturas mentais, o que, tomando por base a epistemologia de Piaget, manteria somente certas características de estágios precedentes, mesmo com o avanço da idade cronológica da criança. Por exemplo, levando em conta o pensamento egocêntrico, característico do início do desenvolvimento do pensamento infantil, dependendo do nível de comprometimento do autismo infantil, a criança, mesmo em idade avançada, pode permanecer com estruturas mentais com predomínio de imagens, ao invés do conceito; ausência de lógica e dificuldade de diferenciar de maneira representativa, o mundo subjetivo do mundo objetivo.

Essas características podem estar relacionadas às dificuldades de atribuição de estados mentais das crianças com autismo apontadas em diversas pesquisas como as de Baron-Cohen, Leslie e Frith (1985); Barros (2008); Fernandes e Mendes (2002); Gallo-Penna (2011); Mainieri (2000); Mattos (1996); Gonçalves e Domingues (2014); Velloso (2011).

\section{MÉTODO}

\section{Participantes}

A amostra foi selecionada pelo critério de conveniência em duas instituições localizadas no estado de São Paulo. Participaram desta pesquisa do tipo exploratória com método de estudo de casos, duas crianças diagnosticadas com autismo, ambas com a idade de dez anos. A pesquisa foi aprovada pelo comitê de Ética da Universidade de São Paulo. Para confiabilidade do diagnóstico de autismo, as crianças foram submetidas às escalas de rastreio de autismo CARS-Br (Pereira, 
Riesgo, \& Wagner, 2008) e ATA (Assumpção Jr., Gabriel, Kuczinski, \& Rocca, 1999), além da apresentação de laudo diagnóstico recente (menos de um ano) assinado por psiquiatra ou neurologista.

A ausência de rebaixamento do Quociente de Inteligência (QI), ou seja, de deficiência intelectual, também foi averiguada na presente investigação, no intuito de evitar interferências nos resultados. Por este motivo, os participantes foram submetidos à aplicação do teste de inteligência R-2 (Alves \& Rosa, 2000); de dois subtestes da escala de inteligência Wechsler infantil, 3. edição - WISC III (Figueiredo, 2002) e da Escala de Comportamento Adaptativo Vineland (Sparrow; Balla, \& Cicchetti, 1984).

Felipe (nome fictício), um dos participantes, apresentou laudo diagnóstico assinado por psiquiatra com o CID-10, F84.0 (Autismo infantil) e alcançou 25 pontos na escala ATA e 32,5 na escala CARS-Br - autismo leve. Demonstrou ausência de deficiência intelectual, com 70 pontos (comportamento adaptativo médio inferior) na Escala de Vineland e QI - percentil 50 (inteligência média). Nos subtestes Cubos e Vocabulários, Felipe obteve pontos ponderados, 6 e 5, respectivamente, o que está um pouco abaixo da média.

A segunda participante, Luiza (nome fictício), apresentou laudo diagnóstico assinado por neuropediatra com o CID 10, F84.5 (Síndrome de Asperger) e alcançou 25 pontos na escala ATA e 32,5 na escala CARS-Br - autismo leve. Demonstrou ausência de deficiência intelectual, com 70 pontos (comportamento adaptativo médio inferior) na Escala de Vineland e QI - percentil 10 (inteligência média inferior). Nos subtestes Cubos e Vocabulários, Luiza obteve pontos ponderados, 8 e 6, respectivamente, o que está dentro da média.

\section{Instrumentos utilizados e procedimentos}

Os procedimentos desta pesquisa resumem-se em:

- Avaliar a teoria da mente dos participantes $(\mathrm{N}=2)$, por meio de tarefa de crença falsa e análise do emprego dos termos mentais.

- Investigar sobre a diferenciação entre a dimensão psicológica e a física (interno X externo) dos participantes de pesquisa, por meio de entrevista clínica piagetiana.

- Investigar sobre a manutenção da atenção compartilhada e a compreensão do contexto da história dos participantes de pesquisa, por meio de contos de fadas e histórias sociais.

As histórias sociais são histórias narradas de forma fragmentada, as quais utilizam recursos visuais como fotografias, desenhos ou pictogramas, envolvendo 
situações de interações sociais que auxiliem na compreensão dos estados mentais próprios e do outro (Gray, 1998; Gray \& Garand, 1993).

\section{RESULTADOS E DISCUSSÃo}

Os dois participantes, Felipe e Luiza, erraram a tarefa de crença falsa, justificando suas respostas com base no não importismo - de acordo com Piaget (1947), quando a criança responde qualquer coisa que lhe vier à mente, sem relação com o que lhe foi perguntado - em ecolalias e na ausência da intenção de comprová-las. Apesar de os participantes errarem a tarefa de crença falsa, empregaram uma quantidade considerável de termos mentais em seus discursos, levando em conta suas condições clínicas.

De acordo com Gonçalves e Souza (2016) a partir da leitura de Leslie (1987; 1988), o emprego de termos mentais evidencia a capacidade de atribuição de estados mentais. Ao contrário, para Piaget (1947) no plano da ação, a presença de termos mentais na linguagem da criança não significa que esta consiga diferenciar, no plano da reflexão, entre os estados mentais e os físicos. A tabela abaixo descrita demonstra a porcentagem de termos mentais pronunciados pelos participantes desta investigação.

Tabela 1 Porcentagem de termos mentais em relação ao total de palavras pronunciados durante as sessões (São Paulo, 2016).

\begin{tabular}{|l|c|c|c|}
\hline \multicolumn{1}{|c|}{ Participante } & Total de palavras & Total de termos mentais & \% Termos mentais \\
\hline Felipe & 1.620 & 65 & $4,01 \%$ \\
\hline Luiza & 1.887 & 101 & $5,35 \%$ \\
\hline
\end{tabular}

Ainda, em relação ao emprego de termos mentais, nota-se na Tabela 2, abaixo descrita, que o maior número de termos mentais pronunciados pelos participantes da presente investigação, refere-se aos termos relacionados às emoções e o menor número se refere aos termos relacionados com a intenção dos personagens.

Cabe aqui destacar que a participante Luiza pronunciou um maior número de termos mentais relacionados às emoções do que Felipe, porém necessitou de apoio por meio das figuras ilustrativas em quase cem por cento das vezes que inferiu sobre estes estados mentais dos mesmos personagens. Diferentemente, o participante Felipe reconheceu as emoções dos personagens das histórias narradas, recorrendo muito pouco ao apoio das figuras ilustrativas. 
Tabela 2 Frequência de termos mentais pronunciados pelos participantes nas sessões em relação ao número de palavras ditas (São Paulo, 2016).

\begin{tabular}{|c|c|c|c|c|c|c|c|}
\hline Participante & Caract. & Crença & Desejo & Emoção & Intenção & $\begin{array}{c}\text { Termos } \\
\text { mentais }\end{array}$ & $\begin{array}{c}\text { Total de } \\
\text { Palavras }\end{array}$ \\
\hline Felipe & 5 & 8 & 5 & 47 & 0 & 65 & 1.620 \\
\hline Luiza & 12 & 17 & 5 & 59 & 8 & 101 & 1.887 \\
\hline
\end{tabular}

Em relação às entrevistas clínicas com método piagetiano, notou-se que os participantes, de maneira geral, estão no primeiro estágio do processo de tomada de consciência em relação ao dualismo entre os aspectos físicos e psicológicos. Por este motivo, Luiza e Felipe apresentam inocência em relação à diferenciação entre o próprio ponto de vista e o ponto de vista do outro, demonstrando alto coeficiente de egocentrismo em suas inteligências representativas. O Quadro 1 demonstra a discussão citada anteriormente.

Quadro 1 Comparação das entrevistas clínicas segundo Piaget.

\begin{tabular}{|c|c|c|c|}
\hline Participante & Pensamentos & Palauras & Sonhos \\
\hline \multirow{2}{*}{$\begin{array}{c}\text { Luiza } \\
\text { (10 anos) }\end{array}$} & $\begin{array}{c}\text { Os pensamentos estão } \\
\text { na cabeça, porém se esta } \\
\text { for aberta, aparecerá uma } \\
\text { lâmpada. }\end{array}$ & $\begin{array}{c}\text { O nome da Luiza está escrito } \\
\text { na Luiza. }\end{array}$ & $\begin{array}{c}\text { Confusão entre o sonho e o } \\
\text { pensamento; entre o sonho } \\
\text { e a realidade. }\end{array}$ \\
\cline { 2 - 4 } & Primeiro estágio & Primeiro estágio & Primeiro estágio \\
\hline \multirow{2}{*}{$\begin{array}{c}\text { Felipe } \\
\text { (10 anos) }\end{array}$} & $\begin{array}{c}\text { Os pensamentos estão na } \\
\text { boca e podem ser tocados. }\end{array}$ & A palavra forte é forte. & $\begin{array}{c}\text { O sonho está na noite e nas } \\
\text { nuvens. }\end{array}$ \\
\cline { 2 - 4 } & Primeiro estágio & Primeiro estágio & Primeiro estágio \\
\hline
\end{tabular}

\section{CONCLUSÕES E IMPLICAÇÕES}

Os resultados desta pesquisa dão indícios de que o emprego dos termos mentais não significou que os participantes diferenciassem entre o psicológico e o físico, pelo desequilíbrio entre assimilação e acomodação. Em outras palavras, a criança, primeiramente, emprega termos mentais em seus discursos predominantemente por assimilação, mas isto não quer dizer que este fato gere, neste momento, a busca de adaptação à realidade. No entanto, à medida que, a partir da estrutura representativa, a intuição dê lugar à construção gradativa da inteligência 
operatória, caracterizada pela reversibilidade de pensamento e início da superação do egocentrismo, o emprego dos termos mentais poderá ser reconstruído no plano da representação por meio também da acomodação.

Além disso, constatou-se que os discursos dos participantes com autismo foram amplamente baseados em ecolalias imediatas e tardias, monólogos coletivos e respostas caracterizadas pelo não importismo e pela fabulação, o que aponta alto coeficiente de egocentrismo correspondente aos discursos encontrados em crianças pequenas (estágio pré-operacional).

Estes apontamentos indicam uma possível relação entre as dificuldades de teoria da mente das crianças com autismo e a tendência destas apresentarem inocência em relação à diferenciação entre o próprio ponto de vista do ponto de vista do outro, fruto de um alto coeficiente de egocentrismo de seus pensamentos.

Por fim, esta pesquisa pode somar-se ao corpo de estudos sobre a relação entre a capacidade de teoria da mente e as principais características dos indivíduos com autismo. A compreensão dos aspectos relevantes do processo da representação mental, da construção subjetiva de si e do outro, refletem a forma peculiar com que a criança com autismo constrói sua subjetividade e, internamente, o mundo real. Esta compreensão pode oportunizar a criação de ferramentas clínicas e pedagógicas, com vistas às interações mais profícuas com estes indivíduos.

\section{REFERÊNCIAS}

Alves, I. C. B., \& Rosa, H. R. (2000). R-2: Teste não verbal de inteligência para criança. Manual. São Paulo, SP: Vetor.

Amorim, L. C. D. (2008). O conceito de morte e a sindrome de Asperger. Dissertação de Mestrado em Psicologia Clínica. 116 f. São Paulo: Universidade de São Paulo, USP. Recuperado de: www.teses.usp. br/teses/disponiveis/47/47133/tde-19022009.../Amorim_me.pdf.

Assumpção Jr., F. B., \& Kuczinski, E. (2011). Diagnóstico diferencial psiquiátrico no autismo infantil. In: J. Schwartzman \& C. Araújo (Orgs.). Transtornos do espectro do autismo. (43-54). São Paulo, SP: Memnom.

Assumpção Jr., F. B., Gabriel, M. R., Kuczinski, E., \& Rocca, C. C. (1999). Escala de avaliação de traços autísticos (ATA): validade e confiabilidade de uma escala para a detecção de condutas autísticas. Arq. Neuropsiquiatria, 57(1), 23-9.

Baron-Cohen, S., Leslie, A., \& Frith, U. (1985). Does the autistic child have a "theory of mind?". Cognition, 21, 37-46.

Barros, P. (2008). Avaliação da empatia em crianças e adolescentes com sindrome de Asperger. Dissertação de Mestrado em Psicologia Social. 94 f. Rio de Janeiro, RJ: Universidade do Estado do Rio de Janeiro - UERJ. Recuperado de: http://livros01.livrosgratis.com.br/cp079527.pdf 
Bosa, C. A., \& Callias, M. (2000). Autismo: breve revisão de diferentes abordagens. Psicologia: Reflexão e Crítica, 13(1). doi: 10.1590/S0102 79722000000100017.

Carraro, L. (2003). A metarrepresentação na brincadeira de faz-de-conta: uma investigação da teoria da mente. Dissertação de Mestrado. 107 f. Porto Alegre, RS: Universidade Federal do Rio Grande do Sul. Rio Grande do Sul. Recuperado de http://www. lume.ufrgs.br/handle/10183/3524.

Domingues, S. F. S. (2006). Teoria da mente: um procedimento de intervenção aplicado em crianças de 3 a 4 anos. Tese de Doutorado em Psicologia da Educação. 138 f. São Paulo: Pontifícia Universidade Católica de São Paulo - PUC.

Dourado, F. (2011). . Autismo e Cérebro Social: Compreensão e Ação. Fortaleza, CE: Premius.

Fernandes, F. D. M., \& Mendes, E. R. R. (2002). Teoria da mente: uma comparação entre autistas, portadoras da síndrome de Asperger e crianças normais. Temas sobre desenvolvimento, 11(61), 25-29.

Figueiredo, V. I. M. (2002). Escala de Inteligência Wechesler para crianças: adaptação e padronização de uma amostra brasileira ( $3^{\text {a }}$ ed.). São Paulo, SP: Casa do Psicólogo.

Gallo-Penna, E.C. (2011). Teoria da mente e autismo: influência da linguagem parental explicativa de estados mentais sobre o desenvolvimento da compreensão social. Tese não publicada (Doutorado em Psicologia da Educação). 139 f. São Paulo, SP: Pontifícia Universidade Católica de São Paulo - PUC.

Golleman, D. (2006). Inteligência Social: o poder das relações humanas. Rio de Janeiro. RJ: Campus.

Gonçalves, P. L., \& Domingues, S. F. S. (2014). A influência das narrativas de histórias infantis em crianças e adolescentes com transtorno do espectro do autismo. Projeto elaborado com o apoio do Programa Institucional de Iniciação Científica da Universidade de Guarulhos, 2014. In: XII Jornada Apoiar: A clínica social propostas, pesquisa e intervenções. Universidade de São Paulo. São Paulo, SP: Vetor Editora Psicopedagógica, 807-820.

Gray, C. (1998). Social Stories and comic strip conversations with students with Asperger syndrome and high-functioning autism. In: E. Schoppler, G. Mesibo \& L. Kunce. (Eds.). Asperger syndrome or high-functioning autism? 1, 167-198. New York: Plenum.

Gray, C., \& Garand, J. D. (1993). Social stories: improving responses of studies with autism with accurate social information. Focus on Autistic Behavior, 8(1), 1-10.

Jou, G. I., \& Sperb, T. M. (1999). Teoria da mente: diferentes abordagens. Psicologia: Reflexão e Crítica, 12(2). doi: org/10.1590/S0102-79721999000200004.

Kanner, L. (1943). Affective disturbances of affective contact. Nervous Child, 217-50.

Klin, A. (2006). Autismo e Síndrome de Asperger: uma visão geral. Revista Brasileira de Psiquiatria, 28 (Supl. I), 3-11.

Leon, V. (2008). A compreensão e a produção de enunciados metafóricos em crianças com transtornos globais do desenvolvimento. Tese de Doutorado e Psicologia. $140 \mathrm{f}$. 
Porto Alegre, RS: Universidade Federal do Rio Grande do Sul - UFRGS. Recuperado de: www.lume.ufrgs.br/bitstream/handle/10183/17822/000723540.pdf.

Leslie, A. M. (1987). Pretense and representation in infancy: the origins of "theory of mind”. Psychological Review, 94(4), 412-426.

Leslie, A. M. (1988). Some implications of pretense for mechanisms underlying the childs' theory of mind. In: J. Astington \& P. Harris, Developing theories of mind ( $\mathrm{p}$. 19-46). New York: Cambridge University Press.

Lillard, A. S. (1993). Pretend play skills and the child's of theory of mind. Child Development, 64, 348-371.

Lima, R. C. (2010). Autismo como transtorno da memória pragmática: teses cognitivistas e fenomenológicas à luz da filosofia de Henri Bergson. Tese não publicada (Doutorado em Saúde Coletiva). 154fls, Rio de Janeiro, RJ: Universidade do Estado do Rio de Janeiro - UERJ.

Mainieri, A. G. (2000). A teoria da mente na vida diária de crianças autistas. Dissertação não publicada (Mestrado em Psicologia do Desenvolvimento). 97fls. Porto Alegre, RS: Universidade Federal do Rio Grande do Sul - UFRGS.

Mattos, M. I. P. (1996). O autismo sob a perspectiva da teoria da mente: na intersecção entre o afeto e a cognição. Dissertação não publicada. (Mestrado em Psicologia). 86fls. Porto Alegre, RS: Universidade Federal do Rio Grande do Sul - UFRGS.

Pereira, A., Riesgo, R. S., \& Wagner, M. B. (2008). Autismo infantil: tradução e validação da Childhood Autism Rating Scale para uso no Brasil. Jornal da Pediatria, 84(6), 487-494.

Perner, J. (1991). Understanding the representational mind. Cambridge: MA: MIT Press.

Piaget, J. (1923). El pensamiento simbólico y el pensamiento del ninõ. (S. Pasternac, Trad.) Genebra: Archives de Psychologie.

Piaget, J. (1947). A representação do mundo na criança: com o concurso de onze colaboradores. (A. U. Sobral, \& M. S. Gonçalves, Trads.) Aparecida, SP: Ideias e Letras, 2005. Originalmente publicado em Paris, 1947.

Rimland, B. (1964). Infantile autism: the syndrome and its implications for a neural theory of behavior. New York: Meredith Publishing Company.

Schwartzman, J. S. (2011). Transtornos do espectro do autismo: conceito e generalidades. In: J.Schwartzman \& C. Araújo (Orgs.). Transtornos do espectro do autismo. p. $37-$ 42. São Paulo, SP: Memnon.

Velloso, R. L. (2011). Avaliação de linguagem e de teoria da mente nos transtornos do espectro do autismo com aplicação do teste Strange Stories traduzido e adaptado para a língua portuguesa. Tese de Doutorado em Distúrbios do Desenvolvimento. 114fls. São Paulo, SP: Universidade Presbiteriana Mackenzie. 
